\title{
ON TRANSVERSE VIBRATIONS OF SHALLOW SPHERICAL SHELLS*
}

\author{
BY \\ MILLARD W. JOHNSON AND ERIC REISSNER \\ Massachusetts Institute of Technology
}

1. Introduction. The present paper is concerned with the frequencies of free vibrations of shallow spherical shells of constant thickness. It has been shown earlier that for vibrations of shallow shells which are primarily transverse a considerable simplification of the problem can be effected by a justified neglect of longitudinal inertia in comparison with transverse inertia [1].

Previous applications of this observation included a study of axi-symmetrical vibrations of spherical shells [2], and a study of inextensional vibrations of general shallow shells [3]. In the present paper we investigate vibrations of shallow spherical shells, without axial symmetry. Appropriate solutions of the differential equations are obtained and these are used to obtain the frequencies of free vibrations of a spherical shell segment (or cap) with free edges, in their dependence on the curvature of the segment and on the number of nodal circles and diameters.

We may summarize certain qualitative aspects of our results as follows. Let $H$ be the height of the apex of the spherical cap above the edge plane of the cap and let $h$ be the wall thickness of the shell. When $H / h=0$ we have Kirchhoff's results for the flat plate. When $H / h$ tends to infinity the frequencies of free vibrations of the cap tend either to a limiting frequency which may be called membrane frequency or they tend to the frequencies of inextensional vibrations which we have previously considered [3]. The membrane frequency is limiting frequency for all vibrations with one or more nodal circles. Its value is independent of the number of nodal radii and of nodal circles provided the latter is not zero. On the other hand, the frequencies of inextensional vibrations are a function of the number of nodal radii and presuppose that the number of nodal circles is zero.

2. Differential equations for free transverse vibrations of shallow spherical shells. The differential equations which are to be solved are of the form

$$
\begin{gathered}
\nabla^{2} \nabla^{2} F-\frac{C}{R} \nabla^{2} w=0, \\
D \nabla^{2} \nabla^{2} w+\frac{1}{R} \nabla^{2} F+\rho h \frac{\partial^{2} w}{\partial t^{2}}=0 .
\end{gathered}
$$

The various quantities occurring in (2.1) and (2.2) have the following significance

$w=$ transverse (axial) displacement,

$F=$ Airy's stress function,

$R$ = radius of middle surface of shell,

$h=$ wall thickness of shell,

$C=E h$, longitudinal stiffness factor,

$E=$ modulus of elasticity,

*Received November 2, 1956. A report on work supported by the Office of Naval Research under Contract NR 064-418 with the Massachusetts Institute of Technology. 
$D=E h^{3} / 12\left(1-\nu^{2}\right)$, bending stiffness factor,

$\nu \quad=$ Poisson's ratio,

$\rho \quad=$ density of shell material,

$\nabla^{2}=()_{r r}+r^{-1}()_{r}+r^{-2}()_{\theta \theta}$, Laplace operator in polar coordinates $r, \theta$.

Stress resultants and couples, in polar coordinate form, are given by the following expressions.

$$
\left.\begin{array}{l}
N_{\theta}=\frac{\partial^{2} F}{\partial r^{2}}, \quad N_{r}=\frac{1}{r} \frac{\partial F}{\partial r}+\frac{1}{r^{2}} \frac{\partial^{2} F}{\partial \theta^{2}} \\
N_{r \theta}=-\frac{\partial}{\partial r}\left(\frac{1}{r} \frac{\partial F}{\partial \theta}\right) \\
V_{r}=-D \frac{\partial \nabla^{2} w}{\partial r}+\frac{1}{r} \frac{\partial M_{r \theta}}{\partial \theta}-\frac{r}{R} N_{r} \\
V_{\theta}=-D \frac{\partial \nabla^{2} w}{r \partial \theta}+\frac{\partial M_{r \theta}}{\partial r}-\frac{r}{R} N_{r \theta}
\end{array}\right\},
$$

Equations (2.1) to (2.5), except for the inertia term $\rho h w_{t \imath}$, are the same as those for problems of statics [4].

3. Solutions for simple harmonic motion. We consider solutions of the form

$$
\left(w, F^{\prime}\right)=\left(\begin{array}{c}
\cos n \theta \\
\sin n \theta
\end{array}\right)\left[w_{n}(r), F_{n}(r)\right] e^{i p t}
$$

Substitution of (3.1) into the differential equations (2.1) and (2.2) leads to the ordinary differential equations

$$
\begin{gathered}
L_{n}^{2} F_{n}-\frac{C}{R} L_{n} w_{n}=0 \\
D L_{n}^{2} w_{n}-\rho h p^{2} w_{n}+\frac{1}{R} L_{n} F_{n}=0
\end{gathered}
$$

where the operator $L_{n}$ is defined by

$$
L_{n}=\frac{d^{2}}{d r^{2}}+\frac{1}{r} \frac{d}{d r}-\frac{n^{2}}{r^{2}}
$$

We may write the solutions of (3.2) and (3.3) as follows

$$
w_{n}=\frac{1}{R D \lambda^{4}} \psi_{n}+\chi_{n}
$$




$$
F_{n}=\left(1+\frac{C}{R^{2} D \lambda^{4}}\right) \psi_{n}^{*}+\frac{C}{R \lambda^{4}} L_{n} \chi_{n}+\phi_{n}
$$

In Eqs. (3.5) and (3.6) we have

$$
\lambda=\left[\frac{1}{D}\left(h \rho p^{2}-\frac{C}{R^{2}}\right)\right]^{1 / 4}
$$

and

$$
\begin{gathered}
\psi_{n}= \begin{cases}C_{1,0}+C_{2,0} \log r, & n=0 \\
C_{1, n} r^{n}+C_{2, n} r^{-n}, & 1 \leq n\end{cases} \\
\phi_{n}= \begin{cases}C_{3,0}+C_{4,0} \log r, & n=0 \\
C_{3, n} r^{n}+C_{4, n} r^{-n}, & 1 \leq n\end{cases} \\
\psi_{n}^{*}=\left\{\begin{array}{l}
\frac{1}{4} C_{1,0} r^{2}+\frac{1}{4} C_{2,0} r^{2}(\log r-1), \quad n=0 \\
\frac{1}{8} C_{1,1} r^{3}+\frac{1}{2} C_{2,1} r \log r, \quad n=1 \\
C_{1, n} \frac{r^{2+n}}{4(n+1)}+C_{2, n} \frac{r^{2-n}}{4(1-n)}, \quad 2 \leq n
\end{array}\right. \\
\chi_{n}=A_{1, n} J_{n}(\lambda r)+A_{2, n} Y_{n}(\lambda r)+A_{3, n} I_{n}(\lambda r)+A_{4, n} K_{n}(\lambda r) .
\end{gathered}
$$

The functions $J_{n}, Y_{n}, I_{n}$ and $K_{n}$ are Bessel functions and modified Bessel functions of order $n$. We note that the argument of these functions is real, as long as the frequency $\boldsymbol{p}$ is greater than a reference frequency which we denote by $p_{\infty}$ and which is given by

$$
p_{\infty}^{2}=\frac{C}{h \rho R^{2}} .
$$

Alternatively, $p_{\infty}$ may be written in terms of the rise $H$ of the shell segment which, from $z=r^{2} / 2 R$, follows in the form $H=a^{2} / 2 R$, where $a$ is the base radius of the shell segment, as

$$
p_{\infty}=2\left(\frac{E}{\rho}\right)^{1 / 2} \frac{H}{a^{2}}
$$

The solutions (3.5) and (3.6) involve eight arbitrary constants for each value of $n$. However, for $n=0$ and $n=1$ only six of these have physical significance. The quantities $C_{3,0}$ and $C_{3,1}$ may be omitted as they do not enter into expressions for displacements and stresses. Furthermore, in order that the displacements $u$ and $v$ in radial and circumferential direction be single valued we must have (see [4] for the case $n=0$ ) the relations

$$
C_{2,0}=0, \quad C_{2,1}=0 \text {. }
$$

We next write resultants and couples in a form which corresponds to (3.1), viz.,

$$
N_{r}=\left\{\begin{array}{l}
\cos n \theta \\
\sin n \theta
\end{array}\right\} e^{i p t} N_{r, n}, \quad \text { etc. }
$$


In this way we shall have in terms of the functions $F_{n}$ and $w_{n}$

$$
\left.\begin{array}{c}
N_{r, n}=\frac{1}{r} \frac{d F_{n}}{d r}-\frac{n^{2}}{r^{2}} F_{n}, \\
\left.N_{\theta, n}=\frac{d^{2} F_{n}}{d r^{2}}, \quad N_{r \theta, n}=\mp \frac{d}{d r}\left(\frac{n}{r} F_{n}\right)\right\} \\
V_{r, n}=-D\left[\frac{d L_{n} w_{n}}{d r}-(1-\nu) \frac{n^{2}}{r} \frac{d}{d r}\left(\frac{w_{n}}{r}\right)\right]-\frac{r}{R} N_{r, n} \\
V_{\theta, n}=-D\left[\mp \frac{n}{r} w_{n} \mp(1-\nu) n \frac{d^{2}}{d r^{2}}\left(\frac{w_{n}}{r}\right)\right]-\frac{r}{R} N_{r \theta, n} \\
M_{r, n}=-D\left[\frac{d^{2} w_{n}}{d r^{2}}+\frac{\nu}{r} \frac{d w_{n}}{d r}-\frac{\nu n^{2}}{r^{2}} w_{n}\right] \\
M_{\theta, n}=-D\left[\frac{1}{r} \frac{d w_{n}}{d r}-\frac{n^{2}}{r^{2}} w_{n}+\nu \frac{d^{2} w_{n}}{d r^{2}}\right] \\
M_{r \theta, n}= \pm(1-\nu) D n \frac{d}{d r}\left(\frac{w_{n}}{r}\right)
\end{array}\right\}
$$

4. Boundary conditions for spherical segment with free edge. In order that the edge $r=a$ be a free edge the following four conditions must be satisfied

$$
\begin{array}{ll}
N_{r, n}(a)=0, & N_{r \theta, n}(a)=0, \\
M_{r, n}(a)=0, & V_{r, n}(a)=0 .
\end{array}
$$

In terms of $F_{n}$ and $w_{n}$ and indicating differentiation with respect to $r$ by primes, these conditions assume the following form

$$
\left.\begin{array}{c}
a F_{n}^{\prime}(a)-n^{2} F_{n}(a)_{i}=0 \\
n\left[a F_{n}^{\prime}(a)-F_{n}(a)\right]=0
\end{array}\right\},
$$

Equations (4.3) may be simplified further, to read

$$
\left.\begin{array}{ll}
F_{0}^{\prime}(a)=0, & a F_{1}^{\prime}(a)=F_{1}(a) \\
F_{n}^{\prime}(a)=0, & F_{n}(a)=0 ; \quad 2 \leq n
\end{array}\right\} .
$$

In addition to boundary conditions for $r=a$ we have regularity conditions for $r=0$. Inspection of (3.5) to (3.11) reveals that these regularity conditions require the vanishing of four of the eight constants of integration in (3.5) and (3.6), namely

$$
C_{2, n}=C_{4, n}=A_{2, n}=A_{4, n}=0 \text {. }
$$

This leaves us with the following expressions for $w_{n}$ and $F_{n}$

$$
w_{n}=\frac{1}{R D \lambda^{4}} C_{1, n} r^{n}+A_{1, n} J_{n}(\lambda r)+A_{3, n} I_{n}(\lambda r),
$$




$$
F_{n}=\left(1+\frac{C}{R^{2} D \lambda^{4}}\right) C_{1, n} \frac{r^{n+2}}{4(n+1)}+C_{3, n} r^{n}+\frac{C}{R \lambda^{2}}\left[-A_{1, n} J_{n}(\lambda r)+A_{3, n} I_{n}(\lambda r)\right] .
$$

An equation determining frequencies of free vibrations is now obtained by substituting the solutions (4.7) and (4.8) in the boundary conditions (4.4) and (4.5). In doing this it is convenient to consider the cases $n=0,1$ separately from the general and more complicated case $2 \leq n$.

5. Rotationally symmetric vibrations and vibrations with one nodal diameter. Substitution of (4.7) and (4.8) into (4.4) and (4.5) for $n=0$ and $n=1$ leads to the following system of simultaneous homogeneous equations for the constants of integration in (4.7) and (4.8)

$$
\left.\begin{array}{r}
A_{1,0}\left[\mu J_{0}(\mu)+(1-\nu) J_{0}^{\prime}(\mu)\right]-A_{3,0}\left[\mu I_{0}(\mu)-(1-\nu) I_{0}^{\prime}(\mu)\right]=0 \\
A_{1,0} J_{0}^{\prime}(\mu)-A_{3,0} I_{0}^{\prime}(\mu)=0 \\
\frac{1}{2}\left[1+\frac{C a^{4}}{R^{2} D \mu^{4}}\right] C_{1,0}+\frac{C}{R \mu}\left[-A_{1,0} J_{0}^{\prime}(\mu)+A_{3,0} I_{0}^{\prime}(\mu)\right]=0
\end{array}\right\}
$$

In these equations the quantity $\mu$ is defined as

$$
\mu=\lambda a=\left[\frac{h \rho a^{4}}{D} p^{2}-\frac{C a^{4}}{D R^{2}}\right]^{1 / 4}
$$

or, with the reference frequency $p_{\infty}$ of Eq. (3.12)

$$
\mu=\left[\frac{h \rho a^{4}}{D}\left(p^{2}-p_{\infty}^{2}\right)\right]^{1 / 4} .
$$

It is noted that the systems (5.1) and (5.2) are such that in each case in order to determine admissible values of $\mu$ it is sufficient to consider the first two equations of the system. These first two equations are the same as the corresponding equations for vibrations of flat plates and accordingly the equation determining possible values of $\mu$ is the same as Kirchhoff's frequency equation for vibrations of circular plates with free edge, with zero or one nodal diameter,

$$
\frac{\mu}{2}\left[\frac{J_{n}(\mu)}{J_{n+1}(\mu)}+\frac{I_{n}(\mu)}{I_{n+1}(\mu)}\right]=1-\nu, \quad n=0,1 .
$$

We note from $\left(5.3^{\prime}\right)$ that values of $p$ larger and smaller than $p_{\infty}$ are accounted for by considering the following ranges of $\mu$.

$$
p \geq p_{\infty}: \mu=\zeta e^{i m \pi / 2} ; \quad \zeta>0, \quad m=0,1,2, \cdots
$$




$$
p \leq p_{\infty}: \mu=\zeta e^{i m \pi / 4} ; \quad \zeta>0, \quad m=1,3,5, \cdots
$$

However, the characteristic equation (5.4) can be shown to possess no solutions of the form (5.6). Also, the substitution of (5.5) into (5.4) leaves (5.4) unchanged so that it suffices to consider only real positive values of $\mu$. We conclude that for $n=0,1$, the frequency $p_{\infty}$ represents the lowest possible frequency of free vibration.

For given $\nu$ Eq. (5.4) is satisfied by an infinite sequence of positive values $\mu=\mu_{n, k}$, $k=1,2,3, \cdots$, where $k$ is the number of nodal circles. Let $p_{n, k}$ be the corresponding frequencies of free vibrations and $p_{n, k}^{(0)}$ the values of these frequencies for the case of flat plates, for which $p_{\infty}=0$. According to $\left(5.3^{\prime}\right)$ we have

$$
p_{n, k}^{(0)}=\mu_{n, k}^{2}\left(\frac{D}{h \rho a^{4}}\right)^{1 / 2}
$$

and

$$
p_{n, k}^{2}=p_{n, k}^{(0) 2}+p_{\infty}^{2} .
$$

We may write (5.8) in the form

$$
\frac{p_{n, k}}{p_{n, k}^{(0)}}=\left[1+\left(\frac{p_{\infty}}{p_{n, k}^{(0)}}\right)^{2}\right]^{1 / 2}
$$

or with

$$
\begin{gathered}
\left(\frac{p_{\infty}}{p_{n, k}^{(0)}}\right)^{2}=\frac{E}{\rho} \frac{4 H^{2}}{a^{4}} \frac{h \rho a^{4}}{D} \frac{1}{\mu_{n, k}^{4}}=\frac{48\left(1-\nu^{2}\right)}{\mu_{n, k}^{4}}\left(\frac{H}{h}\right)^{2}, \\
\frac{p_{n, k}}{p_{n, k}^{(0)}}=\left[1+\frac{48\left(1-\nu^{2}\right)}{\mu_{n, k}^{4}}\right]^{1 / 2} .
\end{gathered}
$$

Known values of $\mu_{n, k}$, for $\nu=1 / 3$ and for $k=1,2,3$, are given in Table 1 . Values of the frequency ratio $p_{n, k} / p_{n, k}^{(0)}$ as a function of $H / h$ may be found in Figs. 2 and 3. These results are new only for $n=1$, the case $n \doteq 0$ having previously been considered in [2]. We note that the modes with no nodal circles $(k=0)$ correspond to rigid-body translations and rotations.

We finally note that the frequency $p_{n, k}$ is equal to $p_{\infty}$ in the limiting case $D=0$, or $H / h=\infty$. In this sense, the frequency $p_{\infty}$ may be designated as the membrane vibration frequency of the shell.

6. Vibrations with two or more nodal radii. Introduction of the solutions (4.7) and (4.8) into the boundary conditions (4.4) and (4.5) leads, when $2 \leq n$, to the following system of simultaneous equations.

$$
\begin{aligned}
\left(1+\frac{\kappa^{4}}{\mu^{4}}\right) \frac{1}{4(n+1)} C_{1, n}^{*}+C_{3, n}^{*}+\frac{1}{\mu^{2}}\left[-A_{1, n} J_{n}(\mu)+A_{3, n} I_{n}(\mu)\right]=0, \\
\left(1+\frac{\kappa^{4}}{\mu^{4}}\right) \frac{n+2}{4(n+1)} C_{1, n}^{*}+n C_{3, n}^{*}+\frac{1}{\mu}\left[-A_{1, n} J_{n}^{\prime}(\mu)+A_{3, n} I_{n}^{\prime}(\mu)\right]=0, \\
(1-\nu) n(n-1) \frac{\kappa^{4}}{\mu^{4}} C_{1, n}^{*}+\left\{\left[(1-\nu) n^{2}-\mu^{2}\right] J_{n}(\mu)-(1-\nu) \mu J_{n}^{\prime}(\mu)\right\} A_{1, n} \\
+\left\{\left[(1-\nu) n^{2}+\mu^{2}\right] I_{n}(\mu)-(1-\nu) \mu I_{n}^{\prime}(\mu)\right\} A_{3, n}=0,
\end{aligned}
$$




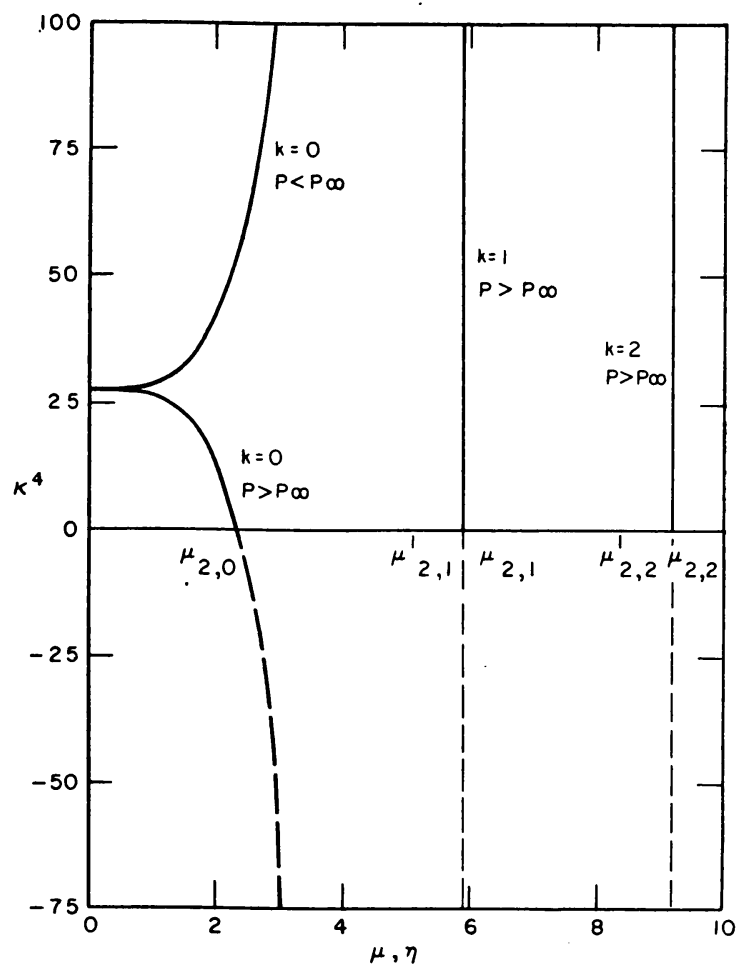

.FIG. 1. Curves $\mu=\mu\left(\kappa^{4}\right)$ and $\eta=\eta\left(\kappa^{4}\right)$ when $\nu=1 / 3$, according to Eqs. (6.7) and (6.13).

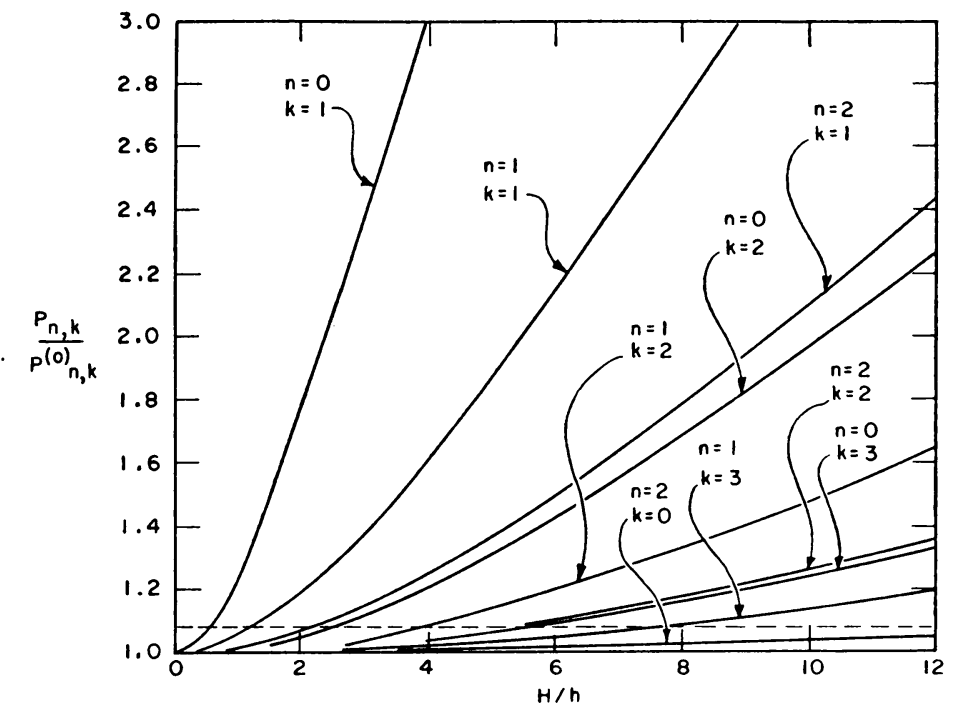

Fig. 2. Values of ratio of shell frequency $p_{n, k}$ to plate frequency $p_{n, k}^{(0)}$ in dependence on ratio of shell rise $H$ to shell thickness $h$ for various values of the number $2 n$ of nodal radii and the number $k$ of nodal circles, for a value $\nu=1 / 3$ of Poisson's ratio. 


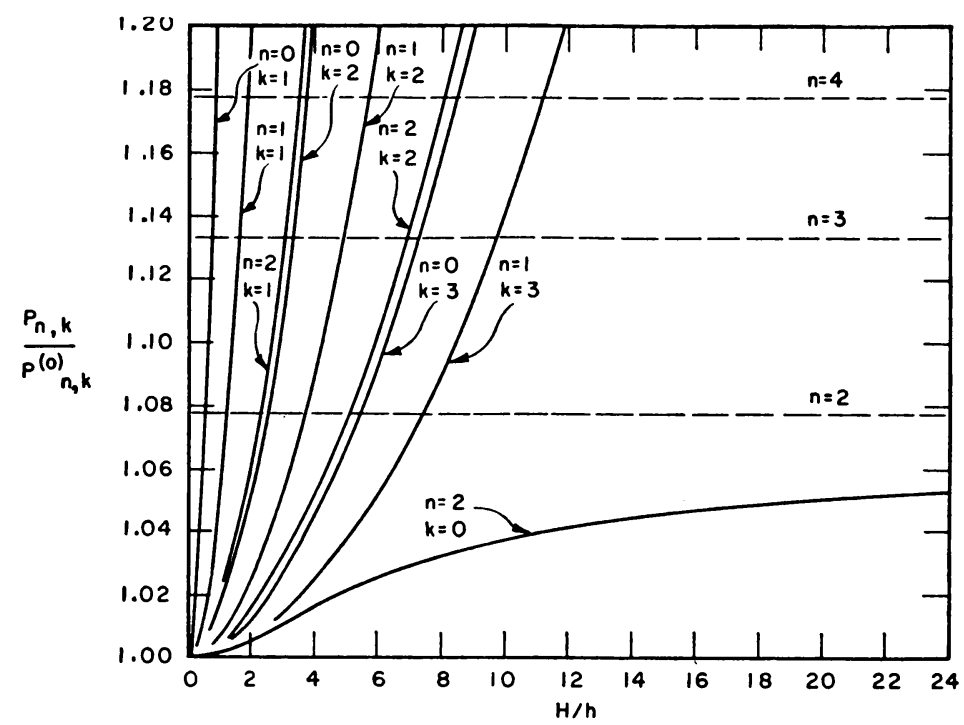

Fig. 3. Enlarged version of part of Fig. 2.

$$
\begin{aligned}
(1-\nu) n^{2}(1-n) \frac{\kappa^{4}}{\mu^{4}} C_{1, n}^{*} & +\left\{(1-\nu) n^{2} J_{n}(\mu)-\left[(1-\nu) n^{2} \mu+\mu^{3}\right] J_{n}^{\prime}(\mu)\right\} A_{1, n} \\
+ & \left\{(1-\nu) n^{2} I_{n}(\mu)-\left[(1-\nu) n^{2} \mu-\mu^{3}\right] I_{n}^{\prime}(\mu)\right\} A_{3, n}=0 .
\end{aligned}
$$

The quantities $C_{m, n}^{*}$ and $\kappa$ are defined as follows.

$$
C_{1, n}=\frac{C}{R a^{n}} C_{1, n}^{*}, \quad C_{3, n}=\frac{C}{R a^{n-2}} C_{3, n}^{*}
$$

and

$$
\kappa^{4}=\frac{C a^{4}}{D R^{2}}=48\left(1-\nu^{2}\right)\left(\frac{H}{h}\right)^{2} .
$$

The vanishing of the determinant of the system (6.1) to (6.4) leads to the following frequency equation

$$
p_{\infty} \leq p: \frac{\mu^{4}}{\kappa^{4}}=\frac{S_{n}(\mu)}{R_{n}(\mu)}-1
$$

where

$$
\begin{aligned}
S_{n}(\mu)=4 n^{2}\left(n^{2}-1\right)(1 & -\nu)\left\{\mu\left[J_{n}(\mu) I_{n}^{\prime}(\mu)-J_{n}^{\prime}(\mu) I_{n}(\mu)\right]\right. \\
& \left.+(n+1)(1-\nu)\left[I_{n}^{\prime}(\mu)-\frac{n}{\mu} I_{n}(\mu)\right]\left[J_{n}^{\prime}(\mu)-\frac{n}{\mu} J_{n}(\mu)\right]\right\}
\end{aligned}
$$

and

$$
\begin{aligned}
& R_{n}(\mu)=\left\{(1-\nu)\left[\mu J_{n}^{\prime}(\mu)-n^{2} J_{n}(\mu)\right]+\mu^{2} J_{n}(\mu)\right\}\left\{(1-\nu) n^{2}\left[\mu I_{n}^{\prime}(\mu)-I_{n}(\mu)\right]-\mu^{3} I_{n}^{\prime}(\mu)\right\} \\
& -\left\{(1-\nu) n^{2}\left[\mu J_{n}^{\prime}(\mu)-J_{n}(\mu)\right]+\mu^{3} J_{n}^{\prime}(\mu)\right\}\left\{(1-\nu)\left[\mu I_{n}^{\prime}(\mu)-n^{2} I_{n}(\mu)\right]-\mu^{2} I_{n}(\mu)\right\} .
\end{aligned}
$$


In order to account for values of $p$ both larger and smaller than $p_{\infty}$ we consider the ranges of $\mu$ given in (5.5) and (5.6). Equation (6.7) is unchanged by the substitution (5.5) so that it will suffice to consider real positive values of $\mu$ for the range $p \geq p_{\infty}$.

$$
p \geq p_{\infty} ; \quad \mu=\left[\frac{h \rho a^{4}}{D}\left(p^{2}-p_{\infty}^{2}\right)\right]^{1 / 4} \geq 0 .
$$

In contrast to the cases $n=0,1$, Eq. (6.7) does possess solutions of the form (5.6), implying that values of $p<p_{\infty}$ are possible for $n \geq 2$. Equation (6.7) remains the same for all $\mu$ of form (5.6) so that it is sufficient to consider the substitution

$$
p \leq p_{\infty} ; \quad \mu=i^{3 / 2} \eta, \quad \eta \geq 0 .
$$

Introducing Kelvin functions of order $n$ by the relations

$$
\left.\begin{array}{l}
J_{n}\left(i^{3 / 2} \eta\right)=\operatorname{ber}_{n} \eta+i \operatorname{bei}_{n} \eta \\
I_{n}\left(i^{3 / 2} \eta\right)=\exp \left(-\frac{n \pi i}{2}\right)\left(\operatorname{ber}_{n} \eta-i \operatorname{bei}_{n} \eta\right)
\end{array}\right\}
$$

we write Eq. (6.7) in the form

where

$$
p \leq p_{\infty} ; \quad \frac{\eta^{4}}{\kappa^{4}}=\frac{U_{n}(\eta)}{T_{n}(\eta)}+1,
$$

$$
\begin{aligned}
U_{n}(\eta)=2(1-\nu) n\left(n^{2}-1\right)\left\{2^{3 / 2} n\right. & \left(\operatorname{ber}_{n}^{\prime} \eta \operatorname{bei}_{n} \eta-\operatorname{ber}_{n} \eta \operatorname{bei}_{n}^{\prime} \eta\right) \\
& +2^{1 / 2}(1-\nu) n(n+1)\left[\frac{n^{2}}{\eta^{2}}\left(\mathrm{ber}_{n}^{2} \eta+\mathrm{bei}_{n}^{2} \eta\right)\right. \\
& \left.\left.-\frac{n}{\eta}\left(\operatorname{ber}_{n}^{2} \eta+\operatorname{bei}_{n}^{2} \eta\right)^{\prime}+\left(\operatorname{ber}_{n}^{\prime} \eta\right)^{2}+\left(\mathrm{bei}_{n}^{\prime} \eta\right)^{2}\right]\right\}
\end{aligned}
$$

and

$$
\begin{aligned}
& T_{n}(\eta)=\left[(1-\nu)^{2} n^{2}\left(n^{2}-1\right)-\eta^{4}\right] 2^{1 / 2} \eta\left(\operatorname{ber}_{n}^{\prime} \eta \operatorname{bei}_{n} \eta-\operatorname{ber}_{n} \eta \operatorname{bei}_{n}^{\prime} \eta\right) \\
&+2^{1 / 2}(1-\nu) \eta^{4}\left\{n^{2}\left[\frac{1}{\eta}\left(\operatorname{ber}_{n}^{2} \eta+\operatorname{bei}_{n}^{2} \eta\right)\right]^{\prime}-\left(\operatorname{ber}_{n}^{\prime} \eta\right)^{2}-\left(\operatorname{bei}_{n}^{\prime} \eta\right)^{2}\right\} .
\end{aligned}
$$

Equations (6.7) and (6.13) assume the same limiting form when $p=p_{\infty}$, that is when $\mu$ and $\eta$, respectively, are zero, namely

$$
\begin{aligned}
p=p_{\infty} ; \quad \kappa^{4}=(1-\nu)(3+\nu) n^{2}\left(n^{2}-1\right) & {\left[1+\frac{1}{4}(1-\nu)(n-2)\right.} \\
& \left.-\frac{n^{2}(n-1)(1-\nu)(4 n+9-\nu)}{16(n+2)^{2}(n+3)}\right]^{-1} .
\end{aligned}
$$

7. Solution of frequency equations for $n=2$. As the character of the frequency calculations is the same for all cases $n \geq 2$ we limit ourselves here to the case $n=2$. According to (6.10) and (6.11) we have that the frequency $p$ is given in the form

$$
p \geq p_{\infty} ; \quad p^{2}=p_{\infty}^{2}+\frac{D}{\rho h a^{4}} \mu^{4},
$$




$$
p \leq p_{\infty} ; \quad p^{2}=p_{\infty}^{2}-\frac{D}{\rho h a^{4}} \eta^{4}
$$

The quantities $\mu$ and $\eta$ are determined as functions of $\kappa^{4}=48\left(1-\nu^{2}\right)(H / h)^{2}$ by solving Eqs. (6.7) and (6.13). We carry out this solution by finding $\kappa^{4}$ as a function of $\mu$ and $\eta$.

Considering first Eq. (6.7) we find that $\kappa^{4}$ has a value slightly above 25 when $\mu=0$ which decreases to zero when $\mu=\mu_{2,0}=2.30$. For larger values of $\mu$, from $\mu=\mu_{2,0}$ to $\mu=\mu_{2,1}^{\prime}$ we have $\kappa^{4}$ negative which means that this part of the curve has no physical significance. From $\mu=\mu_{2,1}^{\prime}$ to $\mu=\mu_{2,1}$ the values of $\kappa^{4}$ are again positive. The next branch of the curve for positive $\kappa^{4}$ lies between the values $\mu_{2,2}^{\prime}$ and $\mu_{2,2}$. The pattern repeats itself with increasing values of $\mu$ (see Fig. 1).

We note that the values $\mu_{2,0}, \mu_{2,1}, \mu_{2,2}$, etc., are roots of Eq. (6.7) for $\kappa^{4}=0$, that is roots of the corresponding flat-plate frequency equation

$$
R_{2}(\mu)=0 \text {. }
$$

The values $\mu_{2,1}^{\prime}, \mu_{2,2}^{\prime}$, etc., are roots of (6.7) for $\kappa^{4}=\infty$, that is roots of the equation

$$
S_{2}(\mu)-R_{2}(\mu)=0 .
$$

We note that for $k=1,2, \cdots$ the values of $\mu_{2, k}$ and of $\mu_{2, k}^{\prime}$ are so nearly the same that for the purpose of frequency calculations we may disregard their difference. This means that for $n=2$ and $k \geq 1$ (and by implication for $n>2$ ) we may take for practical purposes $\mu$ from the flat-plate frequency equation, just as for the cases $n=0$ and $n=1$. However, in addition to the regime in which this is possible we now have the regime $k=0$ where this is not possible. Numerical values of $\mu_{2, k}^{2}$ are given in Table 1 .

We now turn to Eq. (6.13) and determine $\eta$ as a function of $\kappa^{4}$. We find a curve which gives $\kappa^{4}$ somewhat greater than 25 for $\eta=0$, with increasing values of $\kappa^{4}$ as $\eta$ increases. It is apparent that the $\eta\left(\kappa^{4}\right)$-curve represents a continuation of the $\mu\left(\kappa^{4}\right)$-curve for $k=0$. The common point of these two branches for $\mu=\eta=0$ is, as it should be, given by Eq. (6.16).

On the basis of the data contained in Fig. 1 we have calculated the frequency curves $n=2, k=0,1,2$ as contained in Figs. 2 and 3 . It is apparent that there is an essential difference between the case $k=0$ where we have no nodal circles and the cases $k \geq 1$ where we have one or more nodal circles. For $k=0$ the influence of shell curvature remains insignificant, the frequency being very nearly equal to the corresponding flatplate frequency. For $k \geq 1$ the influence of shell curvature is qualitatively significant.

TABLE 1. $\mu_{n, k}^{2}$ for $\nu=1 / 3$.

\begin{tabular}{|c|cccc|}
\hline & $k$ & & & \\
$n$ & 0 & 1 & 2 & 3 \\
\hline 0 & & & & \\
\hline 1 & - & 9.076 & 38.52 & 87.82 \\
2 & 5.251 & 35.24 & 84.38 & 119.0 \\
3 & 12.23 & 52.91 & 112.0 & 193.34 \\
4 & 21.49 & 73.9 & 142.5 & 231.03 \\
\hline
\end{tabular}


8. Frequencies of membrane vibrations. The differential equations of membrane theory follow from (2.1) to (2.5) by setting in them

$$
D=0 \text {. }
$$

If this is done the differential equations (2.1) and (2.2) may be reduced to the following form

$$
\begin{gathered}
\nabla^{2}\left[\rho h \frac{\partial^{2} w}{\partial t^{2}}+\frac{C}{R^{2}} w\right]=0 \\
\frac{1}{R} \nabla^{2} F=-\rho h \frac{\partial^{2} w}{\partial t^{2}}
\end{gathered}
$$

Equation (8.2), with $w=\left\{\begin{array}{c}\cos n \theta \\ \sin n \theta\end{array}\right\} w_{n}(r) e^{i p t}$, is satisfied either when

$$
p^{2}=\frac{C}{R^{2} h \rho}=p_{\infty}^{2}
$$

or, when

$$
\begin{aligned}
& w_{n}=A_{n} r^{n}+B_{n} r^{-n}, \quad 1 \leq n, \\
& w_{0}=A_{0}+B_{0} \log r .
\end{aligned}
$$

In either case $F_{n}$ is found in terms of $w_{n}$ by integrating the relation

$$
L_{n} F_{n}=\rho h R p^{2} w_{n}
$$

Since $D=0$ only the first two of the four boundary conditions (4.1) and (4.2) need to be considered. Appropriate substitution of (8.5) to (8.7) shows that these'equations are satisfied only when $p=0$.

There remains the frequency $p=p_{\infty}$ which is associated with arbitrary functions $w_{n}$, insofar as the differential equation (8.2) is concerned. We have already seen in Sec. 5 that the frequency $p_{\infty}$ is the limiting solution for all modes $n=0,1$, and $k \geq 1$, as $D \rightarrow 0$, or equivalently as $H / h \rightarrow \infty$. For $n \geq 2$ inspection of the solutions (4.7) and (4.8) and of Eqs. (6.1) to (6.4) reveals that the conditions

$$
D \rightarrow 0, \quad \kappa^{4} \rightarrow \infty
$$

are equivalent. Furthermore, for any given mode $k \geq 1, \mu$ as given by (6.7) remains finite as $\kappa^{4} \rightarrow \infty$. Equation (7.1) indicates, therefore, that for modes with one or more nodal circles,

$$
\lim _{D \rightarrow 0} p \rightarrow p_{\infty}, \quad k \geq 1
$$

However, since the one root of (6.13) tends to infinity at the same time that $\kappa^{4} \rightarrow \infty$ we have that the frequency $p_{n, 0}$ given by (7.2) associated with no nodal circles does not tend to the frequency $p_{\infty}$. In the above sense we consider the frequency $p_{\infty}$ as the mem- 
brane vibration frequency of the shallow spherical shell. Figure 4 which represents values of $p_{\infty} / p_{n, k}$ as functions of $H / h$ illustrates the way in which the membrane frequency is approached.

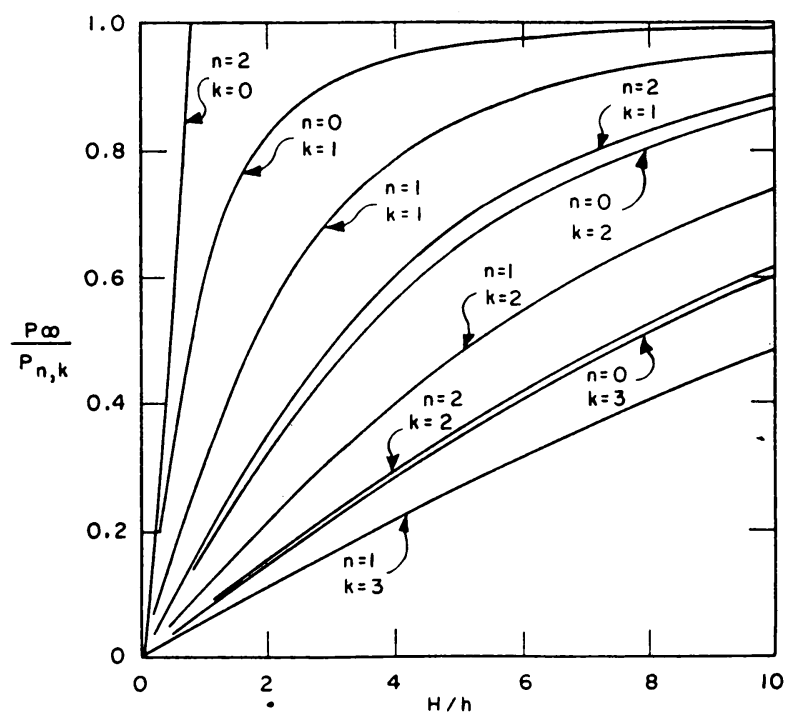

Fig. 4. Relation between shell frequency $p_{n_{\mathrm{o}} k}$ and membrane frequency $p_{\infty}$, when $\nu=1 / 3$.

9. Frequencies of inextensional vibrations. The differential equations for inextensional vibrations are obtained from (2.1) and (2.2) by setting in these equations

$$
\frac{1}{C}=0 \text {. }
$$

It has been shown earlier [3] that inextensional vibrations of shallow spherical shells with free edge can occur only for the modes $k=0, n \geq 2$, with frequency $p_{n}$ given by

$$
\begin{array}{ll}
\frac{H}{h}=0 ; & p_{n}=\left(\frac{D}{\rho h a^{4}}\right)^{1 / 2} \mu_{n, 0}^{2}, \\
\frac{H}{h}>0 ; & p_{n}=\left(\frac{D}{\rho h a^{4}}\right)^{1 / 2}\left[4(1-\nu)\left(n^{2}-1\right) n^{2}\right]^{1 / 2} .
\end{array}
$$

In order to see to what extent these results are contained as limiting cases in our present calculation we note that $1 / C=0$ is equivalent, just as $D=0$ is, to

$$
\kappa^{4}=\infty
$$

provided $H$ is different from zero.

Since when $\kappa^{4} \rightarrow \infty$ we have $p \rightarrow p_{\infty}$ for $k=1,2, \cdots$ it remains to consider what happens when $k=0$. According to Fig. 1 the frequency equation in this case for sufficiently large values of $\kappa^{4}$ is Eq. (6.13). As $\kappa^{4}$ tends to infinity $\eta$ also tends to infinity. We find by means of the asymptotic expansions for Kelvin functions that (6.13) assumes 
the following limiting form for large $\eta$,

$$
\kappa^{4} \simeq \eta^{4}+4(1-\nu)\left(n^{2}-1\right) n^{2} .
$$

Substitution of (9.5) into (7.2) reduces (7.2) to the following form

$$
p^{2} \simeq \frac{D}{\rho h a^{4}} 4(1-\nu)\left(n^{2}-1\right) n^{2}
$$

which shows that when $k=0, H \neq 0$ then when $h \rightarrow 0$ we have $p \rightarrow p_{n}$ as given by (9.3).

On the other hand, for $k=0, h \neq 0$, letting $H \rightarrow 0$ in (7.1) results in $p \rightarrow p_{n}$ as given by (9.2).

The foregoing considerations indicate that our frequency formula, for $k=0$, contains as limiting cases both the appropriate flat-plate frequency formula and the frequency formula for inextensional vibrations of shallow shells. The way in which the transition from one of these formulas to the other takes place is clearly seen by means of the curve which in Figs. 2 and 3 is labeled $n=2, k=0$.

10. The energy of stretching and the energy of bending. Qualitatively, in vibrations which are approximately membrane vibrations the strain energy of stretching will be large compared with the strain energy of bending. On the other hand, for vibrations which are approximately inextensional the strain energy of bending will be large compared with the strain energy of stretching.

We consider in this section the quantitative aspects of the foregoing statement by calculating the ratio

$$
\frac{V_{a}}{V_{b}+V_{s}}
$$

where $V_{\text {c }}$ is the strain energy of stretching and $V_{b}$ is the strain energy of bending. For shallow shells these quantities are given by the following expressions:

$$
\begin{aligned}
& V_{.}=\int_{0}^{a} \int_{0}^{2 \pi} \frac{1}{2 C}\left\{\left(\nabla^{2} F\right)^{2}+2(1+\nu)\right. {\left[\left(\frac{\partial}{\partial r}\left(\frac{1}{r} \frac{\partial F}{\partial \theta}\right)\right)^{2}\right.} \\
&\left.\left.-\frac{\partial^{2} F}{\partial r^{2}}\left(\frac{1}{r} \frac{\partial F}{\partial r}+\frac{1}{r^{2}} \frac{\partial^{2} F}{\partial \theta^{2}}\right)\right]\right\} r d \theta d r \\
& V_{b}=\int_{0}^{a} \int_{0}^{2 \pi} \frac{D}{2}\left\{\left(\nabla^{2} w\right)^{2}+2(1-\nu)\left[\left(\frac{\partial}{\partial r}\left(\frac{1}{r} \frac{\partial w}{\partial \theta}\right)\right)^{2}\right.\right. \\
&\left.\left.-\frac{\partial^{2} w}{\partial r^{2}}\left(\frac{1}{r} \frac{\partial w}{\partial r}+\frac{1}{r^{2}} \frac{\partial^{2} w}{\partial \theta^{2}}\right)\right]\right\} r d \theta d r .
\end{aligned}
$$

We omit the details of the lengthy calculations involved in the evaluation of (10.1) and (10.2). The results of these calculations are incorporated in Fig. 5 which shows how, for $k \geq 1$, the transition from plate to shell is associated with a transfer of strain energy from bending to stretching and how, for $k=0$, we start with bending energy, encounter a certain amount of stretching energy for moderately curved shells, which, with increasing curvature, goes over into bending energy. 


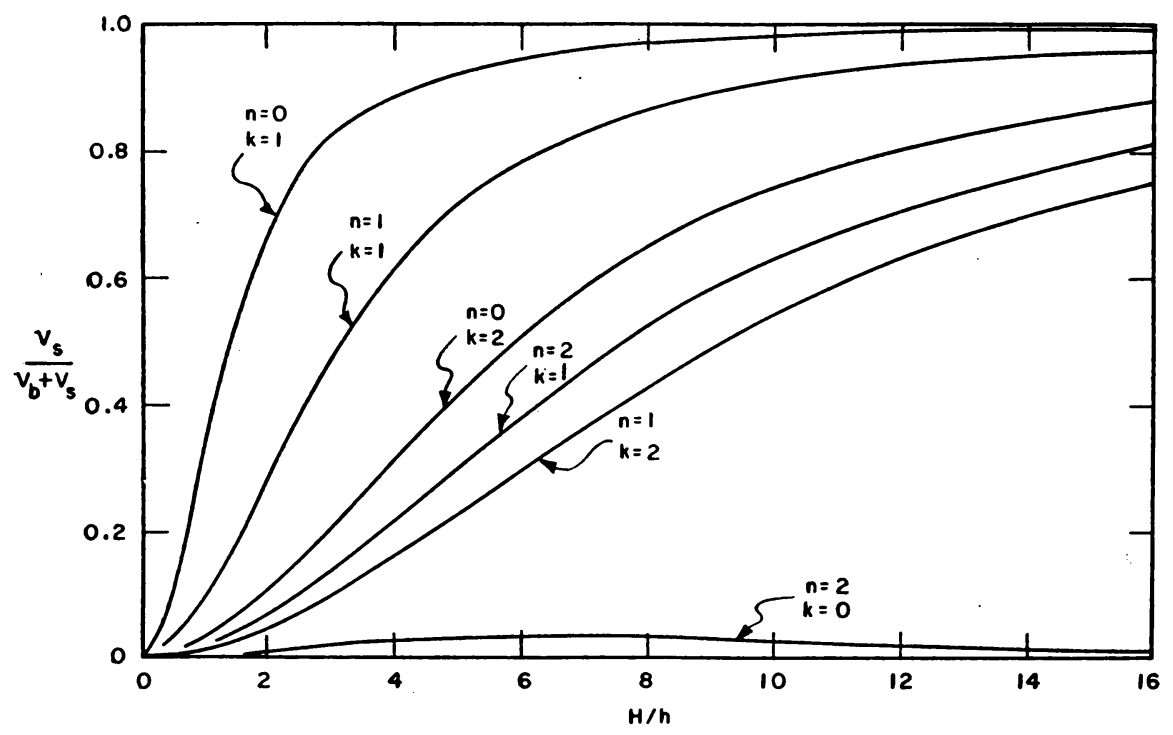

Fra. 5. Ratio of stretching component of strain energy to sum of stretching component and bending component as function of $n, k$ and $H / h$, when $\nu=1 / 3$.

\section{REFERENCES}

[1] E. Reissner, On transverse vibrations of thin shallow elastic shells, Quart. Appl. Math. 13, 169-176 (1955)

[2] E. Reisoner, On axi-symmetrical vibrations of shallow spherical shells, Quart. Appl. Math. 13, 279-290 (1955)

[3] M. W. Johnson and E. Reissner, On inextensional deformations of shallow elastic shells, J. Math. and Phys. 34, 335-346 (1955)

[4] E. Reissner, Stresses and small displacements of shallow spherical shells, II, J. Math. and Phys. 25, 279-300 (1946) 